\title{
Fusion news ignites optimism
}

\author{
News of a 1.3-MJ-output-energy experiment at the National Ignition Facility in the United States in August has \\ raised hopes that laser-based fusion is back on track.
}

$\mathrm{M}$ ore than a decade after the first test firing of all its 192 laser beamlines in early 2009 (Nat. Photon. 3, 177; 2009), confidence is growing that the National Ignition Facility (NIF) in the United States is now close to achieving 'ignition' for laser-triggered nuclear fusion. The accomplishment of ignition self-sustained fusion - would represent not only an important milestone for NIF but also validation that the so-called inertial confinement fusion (ICF) approach can work in practice.

For those not familiar with NIF, it's essentially a giant laser facility, the size of three football fields, which is home to set a of amplified beamlines that boost the power of a neodymium:glass laser to very high levels. Light from these beamlines is then focused symmetrically and simultaneously focused onto a fusion fuel target, in the form a single 1.8 MJ shot that is intended to trigger fusion.

The target consists of a millimetre-sized gold target cylinder called a hohlraum, which houses a pellet of fusion fuel, a mix of deuterium (D) and tritium (T) atoms - two isotopes of hydrogen. The incident laser beams heat the hohlraum causing a strong shock-wave implosion that compresses and heats the DT fuel to extreme temperatures and densities, enabling a plasma of the $\mathrm{D}$ and $\mathrm{T}$ atoms to fuse into helium nuclei, releasing neutrons and energy as part of the process. Importantly, no carbon emissions or greenhouse gases are generated by fusion, making it a clean form of energy generation.

A few months ago, the giant laser facility, which is housed at Lawrence Livermore National Lab in California, announced that it was at the "threshold of fusion ignition" with a laser-shot experiment on 8 August resulting in an output of $1.3 \mathrm{MJ}$ of energy (NIF press release 2021) - the highest yield so far and about 25 times higher than similar experiments conducted in 2018. In the latest tests, the output is estimated to be about $70 \%$ of the laser-shot energy $(1.8 \mathrm{MJ})$, indicating that breakeven - when energy out equals energy in - while not yet reached, is not so far away.

For NIF, and ICF in general, ignition has proved an elusive goal, and a long time coming. This is largely because it is very challenging to meet the necessary extreme conditions for fusion, requiring a near perfect, symmetric implosion of the fuel pellet something that has been harder to achieve

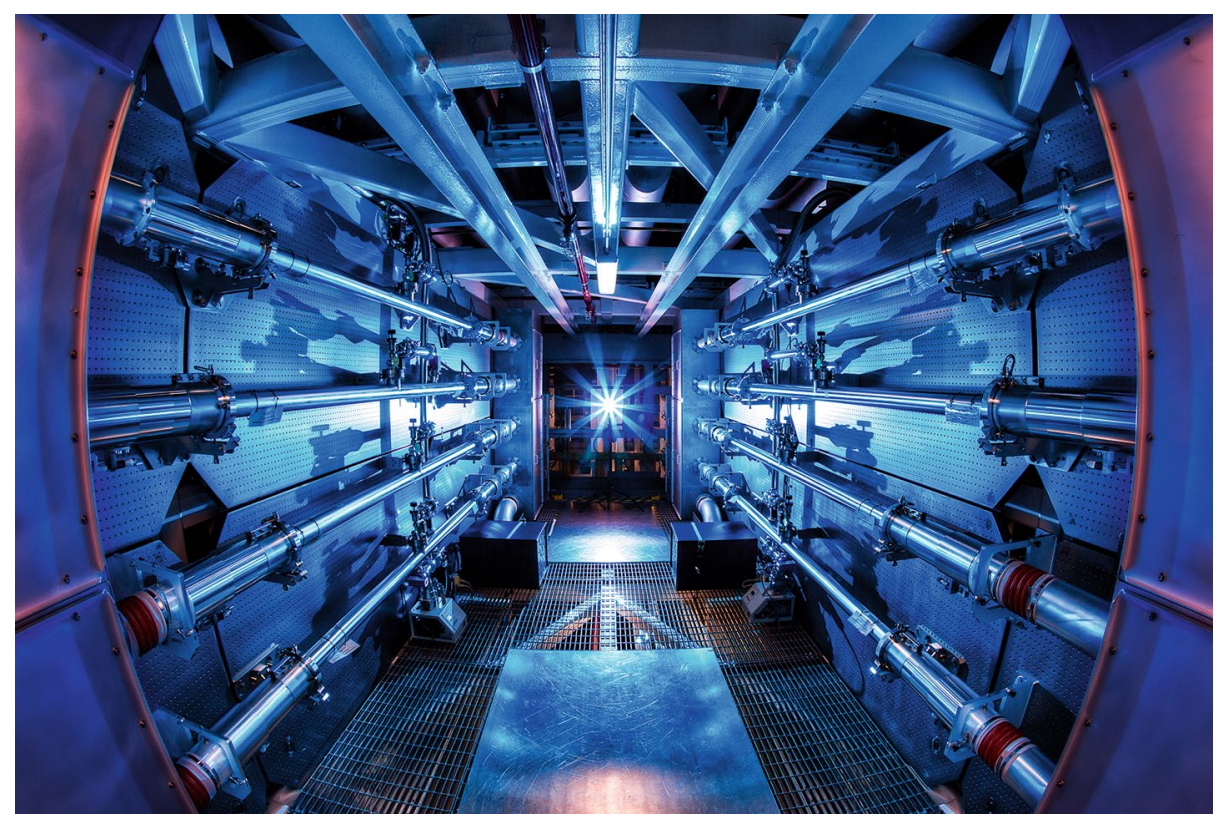

Credit: Damien Jemison / Lawrence Livermore National Laboratory

than first expected due to the presence of instabilities. Hence the excitement in the scientific community over the recent news.

NIF says that the advance has been made possible owing to improvements in diagnostics, target design and fabrication, in particular the hohlraum and fuel capsule shell, and also refinement of the implosion process.

Ignition is the point at which self-sustained fusion takes place with the fusion energy being generated sufficient to combat losses the plasma 'self heats' and burns rather than cooling and fusion ceasing. It's an important precursor for the breakeven point.

While many fusion experts have applauded the recent results as a breakthrough, and undoubtedly the most important achievement of NIF so far, it is ultimately just one step on a very long path to a working fusion reactor.

Perhaps the biggest question now facing the ICF approach is not whether it reaches breakeven - which in itself would be a fantastic feat of science - but whether it can become economic, practical or competitive with other fusion schemes. In particular, approaches based on popular magnetic confinement schemes, such as tokamaks and stellerators, have been receiving lots of investment and innovation in recent years and have progressed.
The international flagship tokamak fusion testbed, ITER in France, is currently under construction and in its main assembly stage. Supported by the European Union, China, India, Japan, Korea, the United States and Russia, it hopes to run its first plasma experiments in 2025 , followed by DT experiments in 2035. It has the ultimate goal of generating an output power that is ten times its input power (500 MW versus $50 \mathrm{MW}$ ).

Furthermore, many private ventures and start-ups are now active in the fusion area, hoping to commercialize the technology. The Fusion Industry Association (FIA), launched in 2018 to act as a collective voice for the industrial development of fusion power, now boasts 25 member companies, including General Fusion, TAE Technologies, Commonwealth Fusion Systems and others. It estimates that over US $\$ 2$ billion has been invested in making fusion power a reality.

It seems that in many ways, the fusion sector is coming of age and now making the transition from being a long-standing scientific dream to becoming an engineering and economic challenge that is attracting increased attention.

Published online: 28 September 2021 https://doi.org/10.1038/s41566-021-00890-Z 